Tools of Biological Research. Edited by Hedley J. B. Atkins, with an Introduction by Sir Cyril Hinshelwood. (Pp. xvi+183; 113 figures. 37s. 6d.) Oxford: Blackwell ; Toronto: Ryerson Press. 1960.

This volume deals with a number of modern research techniques and will therefore be welcomed by a wide variety of specialists in medicine. In his preface the editor explains how, at the meetings of the Surgical Research Society, some senior members found themselves unfamiliar with many of the tools being used in research projects under discussion. Accordingly a two-day symposium was organized at Guy's Hospital, London, on 10 selected "tools of biological research."

On reading this book one becomes more and more impressed by the amount and variety of useful information which it contains. The research worker contemplating a new technique will find the appropriate chapter quite invaluable. In addition to describing the actual technical procedure, its uses and applications are clearly discussed and a very ample selection of references given for further reading.

The introductory address by Sir Cyril Hinshelwood is followed by a most stimulating chapter by Dr. Armitage on the use of statistics in the design of experiments. Fundamental techniques such as "Electron Microscopy," by Dr. Robertson, and "Phase Contrast and Interference Microscopy," by Mr. Barer, are discussed in a way that is most attractive and comprehensible to those with a non-technical training. Clinical pathologists will be interested to read the chapters by Prof. Thompson and Dr. Baker on flame photometry and by Prof. Martin on electrophoresis. Prof. Martin is to be congratulated on his insistence that the basic design of electrophoresis apparatus is of the first importance if reliable results are to be obtained. Pathologists and many others will be fascinated by the excellent chapter on tissue culture by Dr. Honor Fell. As the leading exponent of the subject, and indeed as one of the pioneers in this country, she writes with great clarity in defining the various types of tissue culture, their aims, and their applications. Appropriately, this chapter is followed by a chapter on tissue transplantation by Dr. Brent, who outlines the present achievements and the barriers which at the moment prevent further progress in this technique which may well play a large part in the future of surgery. Medical specialists will be pleased to see a chapter on electromanometry by Dr. Shillingford and one on mass spectrometry by Dr. Hugh Jones. These applications of electronics to the techniques of cardiac catheterization and blood gas analysis have done much to contribute to the enormous advances made in recent years in diseases of the heart and lungs. Radiology has not been neglected, and future improvements are foreshadowed in a lucid account of image intensification by Drs. Combee and Botden.

The book itself is admirably produced, containing diagrams which are clear and illustrations which are of a high quality. The full title of the papers given in the lists of references is a practice which might well be followed by other publishers. It is certain that this work will be widely appreciated, and $\mathrm{Mr}$. Hedley Atkins is greatly to be congratulated on the happy inspiration which resulted in the holding and publication of this symposium. GEORGE CUNNINGHAM.

Diagnostic Radioisotopes. By Charles A. Owen, Ir. (Pp. xix $+425 ; 70$ figures, 49 tables. $£ 6$ 6s.) Oxford: Blackwell. 1959.

A number of competent books on this and related subjects have been published in recent years, and it is against these that this book must be judged. All of them face a common problem: that of achieving a satisfactory balance between, on the one hand, discussion of the purely physical problems of radioactivity measurement, and the theoretical considerations upon which the isotopic techniques are based, and, on the other hand, descriptions of the methods currently in use and interpretation of results. Some succeed better than others in the balance achieved; this book fails completely.

The chapters on counting techniques are largely useless and are in no way improved by the many illustrations of boxes of electronic equipment which have no difficulty in preserving their anonymity behind their front panels. The theoretical bases of some of the problems are poorly explained, others, e.g., those involving multicompartment systems, are not discussed.

The allotment of space to methods and interpretations bears little relation to their importance: for instance, 10 pages are devoted to the problem of $B_{12}$ absorption, but only 13 to the whole of the cardiovascular system, electrolyte and fluid spaces, and protein - metabolism. Indeed, these latter subjects cannot be said to have been properly presented at all.

There are extensive but largely uncritical reviews of the literature relating to medical isotopes, and 64 out of the 381 pages of text are devoted to pointless tables including logarithm tables and that ubiquitous du Bois surface area nomogram.

C. J. HAYTER.

\section{Eight Colloquia on Clinical Pathology}

We regret that in the review of the "Eight Colloquia on Clinical Pathology" (J. clin. Path., 13, 278) we criticized the apparent delay in publication. The review copy was sent direct to the Editor of this journal by the publishers at the request of the Editors of the Colloquia, but they did not point out that the Colloquia had in fact been distributed to the participants within a year of the end of the Congress. We apologize to Professor Welsch and his colleagues for our misunderstanding.-ED. 